\title{
Editorial: Upper and lower limbs: clinical and applied anatomy: from acromial arterial rete to arterial anatomy of toe flaps
}

\author{
Fabrice Duparc ${ }^{1}$ (i) $\cdot$ Bruno Grignon ${ }^{2}$
}

Published online: 17 September 2021

(c) The Author(s), under exclusive licence to Springer-Verlag France SAS, part of Springer Nature 2021

This issue contains some new insights into the anatomy of limbs, with clinical relevance particularly about arteries and flaps, but also about functional anatomy, fibrous structures, muscles and joints innervation. These various articles support the large role of different ways to perform anatomical studies: anatomical dissection, completed by histology, but also 3D-vizualisation and shear wave electrography.

The first part is devoted to the upper limb.

At the junction of the upper arm and the cervical side, crossed by the trans-regional blood supply, the acromial arterial rete is of paramount importance for cervico-humeral flaps. The 3D-visualization of this rete by means of computed tomography scan leads to a better depiction of these flaps design.

Special attention has been paid in this issue on the elbow motion and stability with three articles, respectively, devoted to the biomechanical role of the lacertus fibrosus of the biceps brachii muscle, and to the anconeus muscle and the ulnar collateral ligament as active and passive stabilizers.

Regarding the fibrous structures, the importance of the interosseous membrane is supported by the presentation of the ulnar interosseous tuberosity. All the muscular, tendinous, nervous and vascular structures of the forearm are surrounded but the antebrachial fascia that is exposed through clinical ultrasonography, anatomical and histological findings.

Fabrice Duparc

fabrice.duparc@univ-rouen.fr

Bruno Grignon

b.grignon@chu-nancy.fr

1 Department of Anatomy, Faculty of Medicine-Pharmacy, University of Rouen-Normandy, 22 Boulevard Gambetta, 76183 Rouen, France

2 Department of Anatomy, Faculty of Medicine, University of Lorraine, Avenue de la Forêt de Haye, 54505 Vandœuvre-Lès-Nancy, France
The anatomy of the hand is also detailed in two important studies of great interest for the hand surgeons: the chiasma tendinum and the innervation of the digital joints.

The second part compiles some studies about the lower limb.

The knee is evoked through gracilis, semitendinosus and fascia parameters and their role in hamstring harvesting, the femoral intercondylar notch, and a rare and original presentation of the posterior cruciate ligament.

Three studies deal with the anatomy of the arteries and the clinical applications in the fields of ankle surgery, talar avascular necrosis and toe flaps.

Specific aspects of the ligaments and tendons of the ankle (stiffness of the medial collateral ligament in healthy volunteers, twisted structure of the fetal Achilles tendon) were also investigated by means of shear wave electrography and dissection, respectively.

In both the first and second sections, some rare muscular anatomical variations are shown, that are important for the anatomical culture and for the specialist knowledge, as well as for a better understanding of their possible clinical outcomes.

We wish you an interesting reading.

Publisher's Note Springer Nature remains neutral with regard to jurisdictional claims in published maps and institutional affiliations. 